\title{
Regionalização da Assistência Hospitalar no Sistema Únieo de Saúde: Análise de um objeto de estudo na Saude Coletiva
}

\author{
Regionalization of Hospital Care in \\ Unified Health System: Analysis of an \\ objeet study in public health
}

\section{Rejane Maria Siqueira 1 \\ Rosângela Pereira da Silva ${ }^{2}$ \\ Leonardo Carnut ${ }^{3}$}

\section{Resumo}

Objetivo: Revisar a literatura sobre a Regionalização de Assistência Hospitalar no Sistema Único de Saúde. Metodologia: Foi Utilizado o método de revisão sistemática da literatura acessando a base de dados da BIREME (Biblioteca Virtual em Saúde) a partir do cruzamento dos descritores "SUS", "Assistência Hospitalar" e Regionalização". Resultados: Foram identificados 16 artigos que contemplavam o tema em questão. Os resultados evidenciados nesta revisão afirmam que o desafio maior no processo de regionalização é envolver os secretários de saúde e prefeitos municipais a fim de construir um sistema mais cooperativo e forte na execução de suas atividades. Conclusão: Percebe-se que a regionalização do SUS para de fato acontecer é preciso uma união de interesses políticos, com o estado e os municípios fazendo sua parte, e garantindo a população, ter acesso a cuidados da saúde, à começar pela atenção básica.

\section{Abstraet}

Objectives: To review the literature on the Regionalization of Hospital Care in the Unified Health System Methodology: Used the method of systematic review by accessing the database of BIREME (Virtual Health Library) from crossing the descriptors "SUS", "Hospital Care" and "Regionalization". Results: We identified 16 articles that contemplated the issue at hand. The results shown in this review argue that the biggest challenge in the process of regionalization is to involve the secretaries of health and municipal mayors to build a more cooperative and strong execution of its activities. Conclusion: It is noticed that the regionalization of SUS to actually happen it takes a union of political interests with the state and the municipalities do their thing, and ensuring the population have access to health care, to start with basic care.
1 Graduanda em Gestão Hospitalar e Serviços de Saúde - Faeuldade Santa Emilia - Olinda, Pernambuco, Brasil.

${ }^{2}$ Graduanda em Gestão Hospitalar e Serviços de Saúde - Faeuldade Santa Emilia - Olinda, Pernambuco, Brasil.

3 Mestre em Saúde Coletiva - Faeuldade de Odontologia de Pernambuco/Universidade de Pernambuco [FOP-UPE] - Reeife, Pernambuco, Brasil.

Para correspondência:

Prof. Msc. Leonardo Carnut

email:leonardo.carnut@gmail.com 


\section{Introdução}

A regionalização é a das diretrizes que orientam a organização do SUS definidos pela Constituição Federal Brasileira e pela Lei Orgânica da Saúde. Constituinte do eixo estruturante do Pacto de Gestão do SUS a política de regionalização prevê a formação dos colegiados de gestão regionais que tem a responsabilidade de organizar a rede de ações e serviços de atenção a saúde das populações locais. A regionalização do Sistema Único de Saúde constitui estratégia prioritária do Ministério da Saúde para garantir o direito à saúde, reduzir desigualdades sociais e territoriais; promover a eqüidade e a integralidade da atenção; racionalizar os gastos e otimizar os recursos; e potencializar o processo de descentralização ${ }^{1}$.

Tendo em vista o fortalecimento do processo de regionalização os estados passaram a contar com pólos loco-regionais, os quais formarão a redes estaduais, observando-se os princípios do SUS, tendo como alguma de suas diretrizes: a cooperação, a coresponsabilidade e a autonomia. Estas regiões de saúde são conformadas com base no Plano Diretor para a regionalização da assistência à saúde ${ }^{2}$.

Os procedimentos de alta complexidade mais realizados no SUS, envolvem alta tecnologia e alto custo, objetivando propiciar a população acesso a serviços qualificados, integrando-os aos demais níveis de atenção à saúde (atenção básica de média complexidade) salientando a rede de alta complexidade envolve o enfoque e o planejamento para atender as necessidades ${ }^{3}$.

As principais áreas que compõem a alta complexidade do SUS, organizadas em redes de atenção são: renal crônico oncológico ; cardiovascular; vascular; tráumato-ortopedia; neurologia; otologia; implante coclear; cirurgia das vias aéreas superiores e da região cervical; cirurgia da calota craniana, da face e do sistema estomatognático; transtornos respiratórios do sono; queimaduras; etc ${ }^{3}$.

Além de uma atenção básica totalmente municipalizada, para que a rede de atenção se configure com todos os elementos necessários para a prestação do cuidado, a unidades de Pronto Atendimento (Upas) passaram a integrar a rede estadual de urgência e emergência em janeiro de 2010. O programa foi implantado para preencher a carência de atendimento de emergência de média complexidade no SUS ${ }^{4}$.

Antes pacientes que sofriam pequenos cortes, torções, luxações ou com viroses e febres por exemplos precisavam buscar assistência nas grandes emergências. No entanto, na concepção de rede, cada organização de saúde tem sua missão específica e cabe a cada uma atingir a resolução que lhe é atribuída. Partindo desse pressuposto deve-se implementar mais o acesso a população para pequenos procedimentos em atenção básica e não esse acesso ser diretamente na alta complexidade, tendo como objetivo desafogar os hospitais ${ }^{4}$.

Esse trabalho trata-se de uma revisão sistemática sobre a regionalização da assistência hospitalar no SUS, sua importância para os municípios e regiões e a descentralização na atenção básica em todo Brasil.

\section{Metodologia}

Fez-se uma revisão sistemática da literatura acessando a base de dados da BIREME (Biblioteca Virtual em Saúde - BVS: http://www.bireme.br) a partir do cruzamento dos descritores: "SUS", "ASSISTÊNCIA HOSPITALAR" e "REGIONALIZAÇÃO". A partir desses cruzamentos foram identificados 155 estudos na busca inicial e que depois de passarem pela análise dos revisores (conforme o exposto no fluxograma de identificação dos estudos) restaram 16 artigos que foram incluídos da revisão por serem considerados os artigos que versam sobre o objetivo desse trabalho. Para fins de controle de qualidade, o procedimento de coleta e análise dos estudos foram realizados por dois revisores que obtiveram um nível de concordância considerado aceitável $\left(K_{\text {Kappa }} a_{\text {inter-revisor }}=0,51\right)$. Após identificação dos artigos considerados incluídos na revisão procedeu-se a análise qualitativa dos estudos como demonstrado nos resultados a seguir.

\section{Resultados}

Após as análises dos revisores, percebeuse que 16 artigos contemplavam o objeto da revisão. Estes encontram-se em ordem cronológica de publicação no quadro 01.

O primeiro estudo de Misoczky ${ }^{5}$ trata do desafio de descentralizar com democracia o sistema de saúde através de um plano estratégico para os anos 90, onde foi abordado uma proposta de Distrito Sanitário que permita transformar simultaneamente as relações de poder no setor, o modelo de atenção à saúde e os padrões de gestão. Concluiu-se então que não se trata de estabelecer fluxos rígidos entre os diferentes níveis de atenção, 


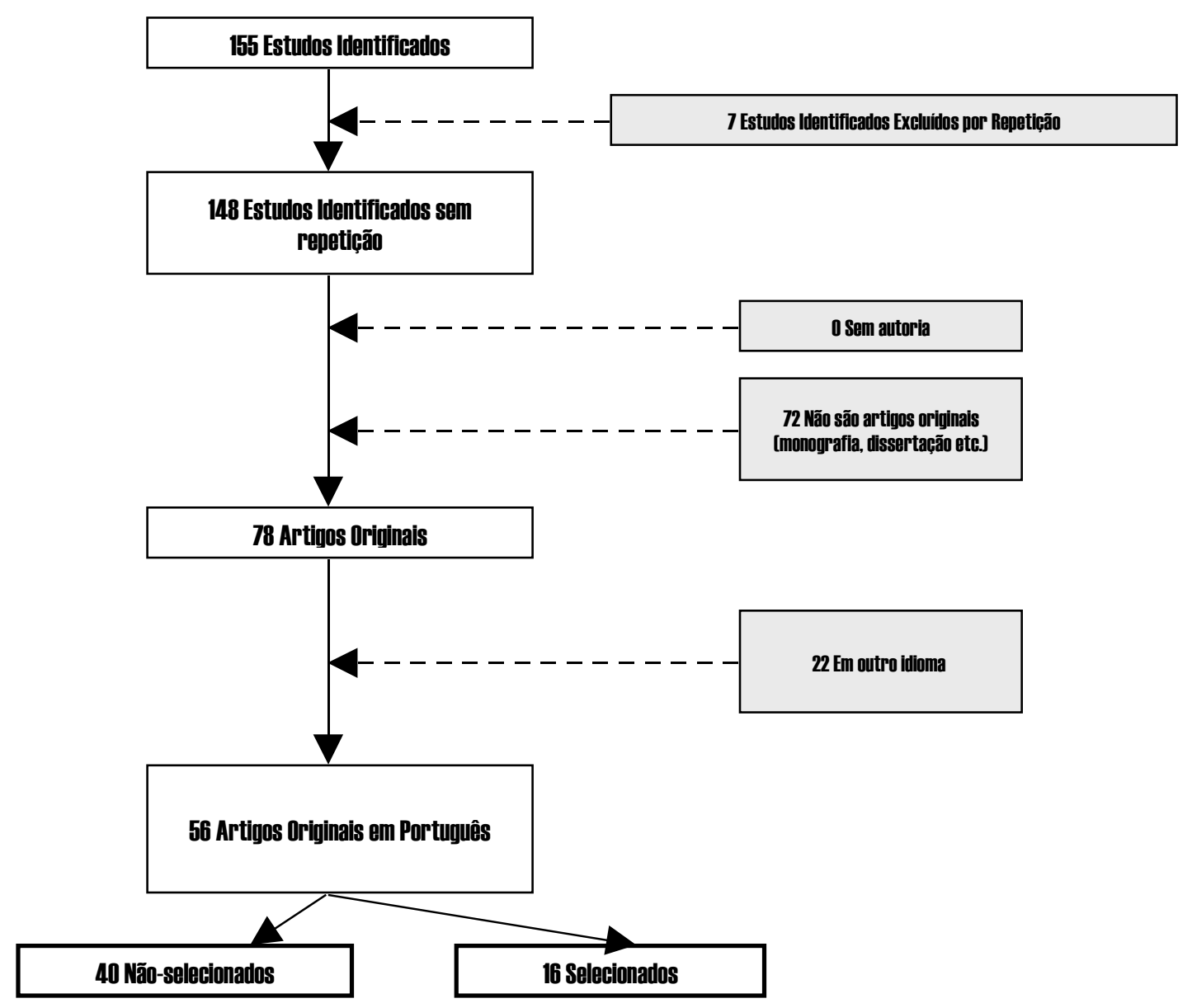

Figura 1. Fluxograma da seleção dos estudos incluídos

mas de garantir a população o acesso à ações e serviços resolutivos e com tecnologia adequada. Para isso é fundamental o apoio de informações epidemiológicas que definam as necessidades de incorporação tecnológica e o perfil das equipes propostas de atenção.

O estudo de Souza ${ }^{6}$ tratou da gestão do processo de descentralização do SUS na década de 1990, relacionando-o a construção e a aplicação das Normas Operacionais Básicas (NOB). O autor relaciona a complexidade e as diferenciações de avanços dos estados e dos municípios, no processo de implantação do SUS. O autor finaliza concluindo que, na época, houve um esgotamento das NOBs para a situação presente da dinâmica descentralização setorial. Sua atenção então já se centrava em outro instrumento: Norma Operacional de Assistência à Saúde.

O estudo de Teixeira ${ }^{7}$ teve como objetivo discutir os limites e possibilidades de implementação de práticas de promoção e vigilância da saúde, levando em conta a proposta de regionalização contida na Norma Operacional da assistência à Saúde (NOAS). Esse estudo aconteceu em todas as regiões e estado do país e sua metodologia foi desenvolvida através de experiências de formação de pessoal e atividades de cooperação técnicas com Secretarias Estaduais 
(SES) e Municipais de Saúde (SMS). Concluiu-se que o desafio maior na implantação do processo de regionalização é envolver os secretários de saúde e prefeitos municipais em um movimento nacional de reorientação do modelo de atenção à saúde que se fundamente na noção de "Municípios Saudáveis" e se desdobrem em ações de promoção a saúde e no fortalecimento das ações de vigilância sanitária, epidemiológica e ambiental. estudo foi realizado na região da Grande Florianópolis. Foram utilizados como metodologia os livros de sala de parto, referente a todos os nascimentos intra-hospitalares e realizado estudo com delineamento transversal e controlado. Conclui-se que os hospitais de maior complexidade atente a maioria dos nascimentos BP e MBP; os hospitais de nível I encaminham suas pacientes para os de maior complexidade.

\begin{tabular}{|c|c|c|c|}
\hline Autores & Título & Revista & Ano \\
\hline Misoczky, M.C. & Distrito sanitário: desafio de descentralizar com democracia & Saúde em Debate & 1991 \\
\hline Souza, R. R. & A regionalização no contexto atual das políticas de saúde & Ciênc. Saúde coletiva & 2001 \\
\hline Teixeira, C. F. & $\begin{array}{l}\text { Promoção e vigilância da saúde no contexto da regionalizaçäo da } \\
\text { assistência à saúde no SUS }\end{array}$ & Cad Saúde Publica & 2002 \\
\hline Paiva, D. B. et al. & Regionalização do atendimento perinatal na Região da Grande Florianópolis & $\begin{array}{l}\text { Arq Catarinense de } \\
\text { Medicina }\end{array}$ & 2003 \\
\hline Guimarães, R.B. & Regiões de saúde e escalas geográficas & Cad Saúde Publica & 2005 \\
\hline Beltrammi, D.G.M. & $\begin{array}{l}\text { Descentralização: o desafio da regionalização para Estados e } \\
\text { Municípios }\end{array}$ & Rev. adm. saúde & 2008 \\
\hline $\begin{array}{l}\text { Viana, A.L.A.; } \\
\text { Ibañez, N. }\end{array}$ & Novas perspectivas para a regionalização da saúde & São Paulo perspect & 2008 \\
\hline $\begin{array}{l}\text { Dubeux, L.S.; } \\
\text { Carvalho, E.F. }\end{array}$ & $\begin{array}{l}\text { Caracterização da oferta de serviços especializados em hospitais de } \\
\text { referência regional: uma contribuição para a regionalização da } \\
\text { assistência à saúde no SUS }\end{array}$ & $\begin{array}{l}\text { Rev. Bras. Saúde } \\
\text { matern. infan }\end{array}$ & 2009 \\
\hline Assis, E. et al. & $\begin{array}{l}\text { Regionalização e novos rumos para o SUS: a experiência de um } \\
\text { colegiado regional }\end{array}$ & Saúde Soc & 2009 \\
\hline Cruz, G.S. & $\begin{array}{l}\text { Gestão compartilhada do curso de qualificação em gestão } \\
\text { regionalizada para o SUS/BA: desafios e possibilidades }\end{array}$ & $\begin{array}{l}\text { R. baiana saúde } \\
\text { pública }\end{array}$ & 2009 \\
\hline Ribeiro, M.S. et al & $\begin{array}{l}\text { A rede de apoio ao SUS em Mato Grosso: uma estratégia para o } \\
\text { fortalecimento do papel do município na regionalização e } \\
\text { implementação do Pacto pela Saúde }\end{array}$ & $\begin{array}{l}\text { Divulg. Saúde } \\
\text { debate }\end{array}$ & 2009 \\
\hline Alves, M.J.M. et al. & $\begin{array}{l}\text { A regionalização e a organização das redes de assistência na macrorregião } \\
\text { sudeste de Minas Gerais: limites e desafios na programação dos serviços para a } \\
\text { assistência ao câncer de mama. }\end{array}$ & APS & 2010 \\
\hline $\begin{array}{l}\text { Reis, Y. A. C.; } \\
\text { Cesse, E.A.P.; } \\
\text { Carvalho, E.F. }\end{array}$ & $\begin{array}{l}\text { Consensos sobre o papel do gestor estadual na regionalização da } \\
\text { assistência à saúde no Sistema Único de Saúde (SUS) }\end{array}$ & $\begin{array}{l}\text { Rev. Bras. Saúde } \\
\text { matern infantil }\end{array}$ & 2010 \\
\hline $\begin{array}{l}\text { Spedo, S.M.; Pinto, } \\
\text { N.R.S.; Tanaka, } \\
\text { O.Y. }\end{array}$ & $\begin{array}{l}\text { A regionalização intramunicipal do Sistema Único de Saúde (SUS): } \\
\text { um estudo de caso do município de São Paulo-SP, Brasil }\end{array}$ & Saúde Soc & 2010 \\
\hline $\begin{array}{l}\text { Pires, M.R.G.M. et } \\
\text { al. }\end{array}$ & $\begin{array}{l}\text { Oferta e demanda por média complexidade/SUS: relação com atenção } \\
\text { básica }\end{array}$ & $\begin{array}{l}\text { Ciênc. Saúde } \\
\text { coletiva; }\end{array}$ & 2010 \\
\hline $\begin{array}{l}\text { Dourado, D.A.; } \\
\text { Elias, P.E.M. }\end{array}$ & $\begin{array}{l}\text { Regionalização e dinâmica política do federalismo sanitário } \\
\text { brasileiro }\end{array}$ & Rev Saúde Publica & 2011 \\
\hline
\end{tabular}

Quadro 1. Descrição dos autores, título, revista e ano de publicação dos estudos incluídos

Já o estudo de Paiva ${ }^{8}$ tratou-se de um trabalho que visou estudar o fluxo de assistência hospitalar aos nascimentos em geral e de risco, baixo peso (BP) e muito baixo peso (MBP) ao nascer. $\mathrm{O}$
No estudo de Ribeiro ${ }^{9}$ teve como objetivo a análise a constituição da rede de apoio ao SUS destacando as práticas de políticas e técnicas no processo de regionalização e implementação do 
Pacto pela saúde. Este estudo foi elaborado no estado do Mato Grosso. Neste contexto a metodologia adotada foi a estratégia de construção da rede de apoio ao SUS um projeto técnico e político, aprovado pela diretoria do COSEMS/MT, sobretudo, com o financiamento do MS. Conclui-se que a conjuntura atual do papel do Estado da coordenação da política estadual com o financiamento do MS, foi determinante para os avanços construídos com gestores municipais de saúde através do seu órgão colegiado, o COSEMS/MT.

Guimarães e Borges ${ }^{10}$ discutiram o processo de regionalização da saúde no Brasil, tendo como base em sua segunda edição (NOAS/ SUS 01/2002). Considerando-se o novo cenário de direcionamento do investimento de unidade pública de saúde, a partir da publicação da norma operacional de assistência à saúde. Para os autores faz-se necessário um esforço de superação da compreensão predominante a respeito de alguns conceitos, principalmente o de região e de escala geográfica. A regionalização da saúde no Brasil é uma necessidade para o fortalecimento do SUS e uma mudança qualitativa da política nacional de saúde. O que está em questão é se a regionalização da saúde brasileira representa ou não um aprimoramento das mediações entre as diversas escalas do SUS.

Pires ${ }^{11}$ investigou a configuração da oferta e demanda por serviços de média complexidade do Distrito Federal (DF) e municípios de Goiás próximo à Brasília. Foi utilizado o método de pesquisa avaliativa, entendida como averiguação em que as pessoas interagem e disputam espaços. Nota-se que a confiança que os usuários têm no hospital do DF e o descrédito na capacidade resolutiva dos centros de saúde, seja do DF ou dos municípios do entorno é um problema a ser superado. Tudo leva a crer que a oferta de atenção básica do DF, apesar de extensa, não tem sido capaz de ampliar o acesso efetivo dos usuários. Recomenda-se a revisão da regionalização e hierarquização dos serviços do DF e Goiás, bem como investimentos na incorporação de tecnologias para atenção básica.

Viana et al ${ }^{12}$ em um ensaio sobre a regionalização, constatou que esta constitui um dos pressupostos da atual fase de descentralização do sistema Único de saúde (SUS) principalmente com o processo de aceleração da redemocratização do País no contexto governamental cooperativo, isto é, Estados e Municípios se unam para elaboração da nova constituição e Organização do poder Democrático. Enfim uma proposta de regionalização da saúde como fato e não como ferramenta deve incorporar, entre outras coisas, as forças e os interesses envolvidos no complexo regional da saúde a fim de construir um sistema mais cooperativo e forte.

Beltrammi et al ${ }^{13}$ objetivaram com seu trabalho, uma análise dos conceitos, das políticas e estratégias desenvolvidas e das expertises acumuladas em vinte anos de construção do Sistema Único de Saúde, a regionalização no contexto dos estados e municípios. Alguns autores sustentam que a regionalização é um movimento dinâmico e flexível que contraria aos métodos fechados e restritos a sua aplicação. Enfim os municípios, por sua vez, devem enfrentar de forma decisiva suas realidades adversas, a fim de conhecer suas reais necessidades a serem compartilhadas no âmbito regional.

Assis e Emerson ${ }^{14}$ trabalharam uma experiência positiva de implantação de um Colegiado Regional de Gestão do estado de São Paulo, foram realizadas várias oficinas e seminários para discutir os pactos pela saúde e a forma de adesão através dos termos de compromissos. A regionalização foi um processo muito rico com intensa participação dos técnicos dos vários municípios. Durante as discussões foram tomadas algumas decisões em que a solidariedade, a vontade de avançar e de resolver os problemas, principalmente de acesso e de garantia de integralidade, sejam os eixos condutores. Foram exemplos a distribuição dos recursos adicionais de média complexidade, a Programação Pactuada e Integrada (PPI) e o PróSanta Casa II. O estado ainda compete com os municípios na execução de atividades em vez de concentrar esforços no que deve ser a sua atribuição.

$\mathrm{Na}$ mesma perspectiva dos autores anteriores, Cruz ${ }^{15}$ em seu estudo objetivou relatar uma experiência de gestão. Tratou-se do curso de gestão regionalizada para o sistema Único de Saúde. A proposta do curso objetivou a construção de capacidades, tecnológicas e a pactuação de alternativas do processo de descentralização do SUS, curso este ocorrido no estado da Bahia através da metodologia compartilhada e coletiva dos saberes com significativo empenho de todo o grupo de gestores. Há avanços que merecem ainda, ser realçada de forma democrática como o grupo tem conduzido os processos decisórios e as relações de trabalho. Com isso a gestão compartilhada desse curso reflete os avanços que se tem buscado implementar no processo de 
educação permanente.

Dubeux et al ${ }^{16}$ estudaram a oferta de serviços especializados em hospitais regionais, considerando os tipos de assistência e os fatores limitantes. Este estudo ocorreu em hospitais regionais de Pernambuco. Para análise utilizou-se o método de avaliação normativa descrito no universo dos Hospitais regionais de Pernambuco, nas regiões de saúde (GERES) do estado. Concluiu-se que esta avaliação demonstrou que a oferta de serviços especializados nos hospitais regionais se distancia da adequação para a assistência hospitalar, conforme preconizado pelo Modelo Estadual de Regionalização da assistência à Saúde adotado.

Spedo e Pinto ${ }^{17}$ estudaram o processo de implementação de um projeto de organização, no entanto ainda há importantes desafios para se caminhar no sentido de alcançar a "imagem objetivo" do SUS. Este estudo se localiza em uma região de saúde do município de São Paulo. O percurso metodológico desse estudo foi fundamentado na pesquisa avaliativa através de uma análise de implementação, centrada em um projeto de intervenção. Com isso o projeto de regionalização proposto para o município de São Paulo apresentava uma racionalidade técnica coerente com os atuais desafios do SUS. Considerou-se estratégica a busca de consensos em torno de grandes objetivos compartilhados no processo respeitando seus interesses particulares. No entanto, deve-se avaliar a potência de um projeto de pactuação dessa natureza restrito apenas ao setor saúde.

Alves et al ${ }^{18}$ procuraram, em seu estudo, identificar as diretrizes de regionalização e financiamento, dando importância ao alerta dos indicadores epidemiológicos e da oferta assistencial, tomando como situação a assistência ao câncer de mama. Esse estudo foi feito em Minas Gerais através de comparações entre a produção de mamografias com o estado do Rio de Janeiro, e uma pesquisa com usuárias em tratamento de câncer de mama em Juiz de Fora - MG. Foram apontados como problema a necessidade de intervenção em aspectos como: a revisão dos limites geográficos, em médio prazo, de um PDI; a implantação do cartão nacional de saúde e do sistema de ressarcimentos aos atendimentos aos usuários de plano de saúde e a revisão dos critérios técnicos.

Reis et al ${ }^{19}$ estudaram os consensos a cerca do papel do gestor estadual na regionalização da assistência à saúde no SUS. Usando como metodologia o modelo teórico-lógico da regionalização da assistência à saúde em desenvolvimento do estado de Pernambuco. O consenso ora estabelecido pode constituir um referencial de elevada utilidade para a pesquisas, podendo servir de base para investigações avaliativas sobre à implantação dos processos de regionalização. Ele representa apenas um consenso provisório sobre o papel do gestor estadual, pode servir como um importante instrumento, orientando sobre os processos chaves que devem ser implementados.

Por fim, Dourado e Elias ${ }^{20}$, em seu trabalho, procuraram examinar as implicações da estrutura Federativa Brasileira no processo de regionalização do SUS na atual conjuntura histórica, em que os estados têm um papel preponderante de promover o bem-estar social. $\mathrm{O}$ estudo ocorre em todo território brasileiro. $\mathrm{O}$ método utilizado foi um estudo de revisão sistemática no caso de cada região. Enfim depois de efetivada a descentralização no SUS, a estrutura municipalizada não era capaz de oferecer realizações do sistema nacional de saúde o que caracteriza a Federação Brasileira.

\section{Disenssão}

A literatura científica relacionada a regionalização da assistência hospitalar demonstrou-se escassa. Tendo em vista que a oferta da atenção básica, apesar de extensa, não tem sido capaz de ampliar o acesso efetivo dos usuários, precisando então investir mais na incorporação de tecnologias para atenção básica. A regionalização da saúde no Brasil é uma necessidade para o fortalecimento do SUS como colocam alguns autores ${ }^{21,22}$ então se faz necessário uma ampliação dos serviços de atenção básica para favorecer, de acordo com os princípios de rede de atenção ${ }^{23} \mathrm{o}$ acesso a atenção hospitalar naqueles municípios que não tem estrutura para mantê-los.

Devido ao não comprometimento dos responsáveis políticos e a falta de recursos, que definam as necessidades de cada região, é fundamental o apoio dos gestores que devido à interesses políticos não colocam em prática as Normas Operacionais Básicas (NOB), para um bom atendimento no SUS, e garantir à população o acesso à ações e serviços resolutivos e adequados. Essa característica da regionalização da assistência foi marcante nos estudo analisados, demonstrando o entrave existente na implantação da diretriz do SUS, fatores que transcendem a questão técnica e 
invadem a seara política na discussão de redes de atenção ${ }^{23}$.

A escala geográfica tem como importância considerar as prioridades assistenciais de cada estado, subdividido em regiões e microrregiões, definidos no plano diretor de regionalização da saúde (PDR). Os módulos assistenciais de microrregional dito por essa normas operacionais do Ministério da Saúde (MS) devem ser organizados e capacitados para atender sua própria população dos municípios a ele adscritos. Essa tema de estudo também gozou de relativa importância como objeto de estudo dos autores que trabalharam a questão da regionalização da assistência hospitalar.

Como a incapacidade resolutiva detectada nos estudos, geram demandas para média e alta complexidade, faz-se necessário um esforço de superação dos envolvidos na compreensão predominante a respeito de alguns conceitos; estados e municípios precisam se unir para elaboração de nova organização do poder democrático; A revisão dos limites geográficos; a implantação do cartão nacional de saúde e a revisão dos critérios técnicos, essas seriam algumas das possibilidades que faria esse sistema funcionar, e claro o trabalho sério dos implementadores de políticas de regionalização diante a essa necessidade de se regionalizar na saúde para toda população.

Não obstante, é importante considerar, durante a análise dos dados revisados, as limitações que o presente estudo apresentou. Esta revisão não foi ampla o suficiente para captar estudos em outros idiomas, detendo-se apenas aos estudos publicados em língua portuguesa. A escolha por essa conduta partiu da decisão de concentrar a busca em estudos sobre a regionalização da assistência hospitalar no Sistema Único de Saúde o que restringe o escopo deste trabalho aos estudos em português apenas. Além disso, admitiu-se um viés de banco dados. Estudos de revisão sistemática devem levar em consideração todos os bancos de dados disponíveis no âmbito de seu objeto de pesquisa, no entanto nesse estudo optou-se por trabalhar apenas como a MEDLINE e SCIELO por se tratarem de ferramentas de acesso gratuito. Por fim, obviamente por se tratar de uma revisão sistemática conceitual (ou teórica), não se aplica nesse estudo a discussão sobre a evidência obtida sobre o objeto.

Contudo é interessante salientar os elementos que conferem validade a este trabalho. A revisão sistemática sobre a temática é importante, pois, como visto nos estudos, apesar de normatiza encontra sério desafios no seu estabelecimento contudo tem sido observado na literatura que este tema tem sido alvo de estudos científicos, comprovando a preocupação da comunidade científica como tema em questão. Adicionalmente, o controle de dados através dos níveis de concordância entre revisores foram estimados dentro dos graus de confiabilidade adequados.

\section{Conclusões}

De acordo com os achados desta revisão conclui-se que há uma escassez de estudos sobre o tema da regionalização da assistência hospitalar e a mesma ainda carece de mais interesse como objeto de estudo da saúde coletiva. No entanto alguns estudos já denunciam algumas lacunas na implementação da regionalização desse nível de atenção como a superação da compreensão predominante a respeito de alguns conceitos; a necessidade de união entre estados e municípios para elaboração de nova organização do poder democrático; a revisão dos limites geográficos; a implantação do cartão nacional de saúde e a revisão dos critérios técnicos sobre a operacionalização da regionalização.

\section{Referêneias}

1. Gil AC et al. Regionalização da saúde e consciência regional. Hygeia. Revista Brasileira de Geografia Médica e da Saúde 2006; 2(3):35-46.

2. Ribeiro $\mathrm{CM}$ et al. Regionalização em saúde e procedimentos hospitalares: uma abordagem metodológica. Rev Paran Desenvolvimento 2005; 109:81-118.

3. Viana et al. Atenção de alta complexidade no SUS: desigualdades no acesso e no financiamento - Projeto Economia da Saúde. Volume I Ministério da Saúde SCTIE/DES .Brasília, DF, Fevereiro, 2005. 153p.

4. O'Dwyer G. A gestão da atenção às urgências e o protagonismo federal. Ciênc saúde coletiva 2010; 15(5):2395-2404.

5. Misoczky MC. Redes e hierarquias: uma reflexão sobre arranjos de gestão na busca em saúde. Rev Adm Pública 2003; 37(2): 335-54.

6. Souza RA. A Regionalização no contexto atual das políticas de saúde. Ciênc saúde coletiva 2001; 6(2): 451-455.

7. Teixeira CF. Promoção da vigilância da saúde no contexto da regionalização da assistência à saúde no SUS. Cad saúde pública 2002; 18(supl.): 153-162.

8. Paiva DB et al. Regionalização do atendimento perinatal na região da grande Florianópolis. Arq. Catarin. Med 2003; 32(3):43-49.

9. Ribeiro MS et al. A rede de apoio ao SUS em mato grosso: uma estratégia para o fortalecimento do papel do município na regionalização e implementação do pacto pela saúde. Divulg. saúde debate 2009; (44): 62-73. 
10. Guimarães RB. Regiões de saúde em escala geográficas. Cad saúde pública 2005; 21(4)1017-1025.

11. Pires MRG et al. Oferta e demanda por média complexidade/SUS: relação com atenção básica. Ciênc Saúde coletiva 2010; 15(supl.1): 1009-1019.

12. Viana ALA, Ibañez $N$. Novas perspectivas para a regionalização da saúde. São Paulo perspect 2008; 22(1): 92-106.

13. Beltramini DGM et al. Descentralização: o desafio da regionaliação para estados e municípios. Rev adm saúde. 2008; 10(41):159-163.

14. Assis et al. Regionalização e novos rumos para o SUS: a experiência de um colegiado regional. Saúde soc 2009; 18(supl.1): $17-21$.

15. Cruz, GS. Gestão compartilhada do curso de qualificação em gestão regionalizada para o SUS/BA: desafios e possibilidades. Rev. baiana saúde pública 2009; 33(1): 6874.

16. Dubeux LS, Carvalho EF. Caracterização da oferta de serviços especializados em hospitais de referências regional. Rev bras saúde matern infat 2009; 9(4): 467-476.

17. Spedo OSM et al. A Regionalização intramunicipal do sistema único de saúde (SUS): um estudo de caso do município de São Paulo - SP, Brasil. Saúde soc 2010; 19(3): 533-546.

18. Alves MJ et al. A regionalização e a organização das redes de assistência na macrorregião sudeste de minas gerais: limites e desafios na programação dos serviços para a assistência ao câncer de mama. Rev APS 2010; 13(supl.1):S26-S34.

19. Reis YA et al. Consensos sobre o papel do gestor estadual na regionalização da assistência à saúde no sistema único de saúde SUS. Rev bras saúde matern infant 2010; 10(supl.1): S157-S172.

20. Dourado DA et al. Regionalização e dinâmica política do federalismo sanitário brasileiro. Rev saúde pública 2011; 45(1): 204-211.

21. Carvalho BG et al. A organização do sistema de saúde no Brasil. Bases da Saúde Coletiva. 2 ed. Andrade, SM; Soares, DA; Cordoni Jr, L (org.), No prelo. 2006.

22. Bodstein R. Atenção básica na agenda da saúde. Ciênc saúde coletiva 2002; 7(3)401-412.

23. Mendes EV. Redes de Atenção à Saúde. Ciênc saúde coletiva 2010; 15(5):2297-2305. 